\title{
Microbe-associated molecular pattern-induced calcium signaling requires the receptor-like cytoplasmic kinases, PBL1 and BIK1
}

\author{
Stefanie Ranf ${ }^{1,2}$, Lennart Eschen-Lippold ${ }^{1}$, Katja Frhlich ${ }^{1}$, Lore Westphal ${ }^{1}$, Dierk Scheel ${ }^{1}$ and Justin Lee ${ }^{1 *}$
}

\begin{abstract}
Background: Plant perception of conserved microbe-derived or damage-derived molecules (so-called microbe- or damage-associated molecular patterns, MAMPs or DAMPs, respectively) triggers cellular signaling cascades to initiate counteracting defence responses. Using MAMP-induced rise in cellular calcium levels as one of the earliest biochemical readouts, we initiated a genetic screen for components involved in early MAMP signaling in Arabidopsis thaliana.

Results: We characterized here the changed calcium elevation 5 (cce5) mutant, where five allelic cce5 mutants were isolated. They all show reduced calcium levels after elicitation with peptides representing bacteria-derived MAMPs (flg22 and elf18) and endogenous DAMP (AtPep1), but a normal response to chitin octamers. Mapping, sequencing of the mutated locus and complementation studies revealed CCE5 to encode the receptor-like cytoplasmic kinase (RLCK), avrPphB sensitive 1-like 1 (PBL1). Kinase activities of PBL1 derived from three of the cce5 alleles are abrogated in vivo. Validation with T-DNA mutants revealed that, besides PBL1, another RLCK, Botrytis-induced kinase 1 (BIK1), is also required for MAMP/DAMP-induced calcium elevations.
\end{abstract}

Conclusions: Hence, PBL1 and BIK1 (but not two related RLCKS, PBS1 and PBL2) are required for MAMP/DAMP-induced calcium signaling. It remains to be investigated if the many other RLCKs encoded in the Arabidopsis genome affect early calcium signal transduction perhaps in dependence on the type of MAMP/DAMP ligands. A future challenge would be to identify the substrates of these various RLCKs, in order to elucidate their signaling role between the receptor complexes at the plasma membrane and downstream cellular signaling components.

Keywords: Calcium, Changed calcium elevation (cce) mutants, Receptor-like cytoplasmic kinase, Signaling

\section{Background}

During their infection attempt, microbes activate intracellular signaling cascades in their potential host. Specific pattern-recognition receptors (PRRs) from the host recognize conserved microbe-associated molecular patterns (MAMPs) or certain signature molecules resulting from tissue damage, often designated as damage-associated molecular patterns (DAMPs) [1]. PRRs are typically receptor-like kinases (RLKs), such as FLS2 (Flagellin-Sensing 2), EFR (Elongation Factor Tu Receptor) or PEPR1/PEPR2 (AtPep-Receptor 1/2). These recognize the MAMPs, flg22 (N-terminal flagellin-derived peptide), elf18 (N-terminal fragment of

\footnotetext{
*Correspondence: jlee@ipb-halle.de

${ }^{1}$ Stress and Developmental Biology, Leibniz Institute of Plant Biochemistry,

Weinberg 3, Halle/Saale D-06120, Germany

Full list of author information is available at the end of the article
}

Elongation Factor Tu) and the DAMP, AtPep1, respectively [2]. Upon binding of the respective ligand [3-5], FLS2, PEPR1/PEPR2 or EFR hetero-oligomerize with BAK1 (BRI1-Associated Kinase 1), a kinase originally found as an interactor of the brassinosteroid hormone receptor, BRI1 [6]. Recent structural studies indicate that BAK1 is also in direct contact with the C-terminal part of the FLS2-bound flg22, and may thus be considered a co-receptor [7]. Accordingly, bak1 mutants are impaired in responses to these MAMPs/DAMPs [5,8,9]. Thus, BAK1 acts as protein partner (or co-receptor?) for multiple pathways in plant immunity and development [10]. On the other hand, signaling induced by other MAMPs, such as chitin is independent of BAK1 [11]. This difference may be a consequence of the different structure of the potential receptor(s) required for perceiving chitin, CERK1 (Chitin Elicitor Receptor Kinase 1), a LysM-containing RLK in Arabidopsis [12-14] as 
compared to the LRR-type RLKs such as FLS2, EFR or PEPR1/R2.

Among the earliest signaling events after MAMP/ DAMP perception are ion fluxes across the plasma membrane including influx of calcium into the cytosol [8,15-17]. The elevation of cytosolic calcium is detected by a number of calcium-binding decoder proteins such as calmodulins or calcium-dependent protein kinases (CPKs) or Calcineurin B-like $(\mathrm{CBL})$ proteins and their partners, CBL-interacting protein kinases (CIPKs) to further transmit the signal $[18,19]$. Calcium, as a signaling molecule, is a prerequisite for most downstream responses elicited by MAMPs/DAMPs. For instance, production of reactive oxygen species (ROS) by the NADPH oxidase, RBOHD, in Arabidopsis [20] is a calcium-dependent process stimulated by direct binding of calcium to EF-hands in the Nterminus of RBOHD. Furthermore, the calciumdependent protein kinase 5 (CPK5) phosphorylates RBOHD to promote its activity [21]. Activation of mitogen-activated protein kinases (MAPKs) also requires calcium since depletion of extracellular calcium or inhibition of calcium channels block MAMP-induced MAPK activation $[9,22]$.

The importance of calcium for plant immunity is also indirectly supported by the observation that phytopathogenic bacteria secrete extracellular polysaccharides to sequester apoplastic calcium and attenuate host MAMP signaling [23]. However, much of plant calcium signaling remains to be discovered, in particular, the steps between perception of MAMPs/DAMPs and generation of the calcium signals. We used an apoaequorin-expressing transgenic Arabidopsis thaliana line to investigate MAMP signaling events in whole seedlings [9]. Aequorin is a calcium sensitive reporter for measuring changes in cellular calcium levels [24]. Upon binding calcium, it oxidizes the bound coelenterazine prosthetic group into excited coelenteramide, which emits blue light at $469 \mathrm{~nm}$. The so-called $\mathrm{L} / \mathrm{L}_{\max }$ ratio of the aequorin-generated luminescence $(\mathrm{L})$ to the total remaining aequorin $\left(\mathrm{L}_{\max }\right)$ is used as an estimate of relative calcium levels. With the appropriate calibration parameters, it is also possible to convert the $\mathrm{L} / \mathrm{L}_{\max }$ values into absolute cytosolic calcium concentrations [25].

We previously demonstrated that the aequorin-based measurement is amenable to high throughput screening and used it to isolate mutants with a changed calcium elevation (cce) phenotype after flg22 elicitation. The first sets of identified cce mutants were the FLS2 receptor and its partner kinase, BAK1 [26]. These mutants represent proof-of-principle of the suitability of the screen in finding signaling components between ligand recognition and calcium flux. This current work reports the characterization of the cce5 mutant and the identification of the receptorlike cytoplasmic kinase (RLCK), PBS1-like 1 (PBL1) being the CCE5 gene, where PBS1 stands for avrPphB sensitive
1, an RLCK targeted by the Pseudomonas syringae pv. phaseolicola protease avrPphB [27]. The analysis of mutants of three related RLCKs revealed an additional requirement of Botrytis-induced kinase 1 (BIK1) for the MAMP/DAMP-induced calcium elevation.

\section{Results}

The changed calcium elevation 5 (cce5) mutant is affected in early signaling

Four other independently isolated changed calcium elevation (cce) mutants did not restore a normal calcium response to flg22 in the $\mathrm{F} 1$ generation when crossed to the previously described cce5 mutant [26] (data not shown). The lack of complementation suggests that these five cce mutants are allelic and thus designated as cce5-1 to cce5-5. All five cce5 mutant lines show a reduced flg22- and elf18induced calcium rise compared to the parental HVA1 line; however, the reduction in the elf18-induced calcium levels appears to be stronger than with flg22 (Figure 1A, B). Correspondingly, elf18-induced MAPK activation was partially reduced and delayed (Figure 1C). Surprisingly, the reduction in flg22-induced MAPK activation was not as obvious as for elf18. It was only visible if a lower concentration (e.g. $10 \mathrm{nM}$ ) of the flg22 peptide was used; at higher concentrations, no difference in comparison to HVA1 was discernible (Additional file 1: Figure S1). Thus, CCE5 may have different signaling role(s) for these two MAMPs. Similarly, other rapid responses such as reactive oxygen species (ROS) accumulation was also reduced for the cce 5 alleles, when treated with flg22 or elf18 (Additional file 1: Figure S2). Since MAPK activation and ROS accumulation occur within minutes upon elicitation, cce5 is mutated in some early signaling component(s).

\section{Differential MAMP/DAMP response of the cce5 mutants is reminiscent of a BAK1-dependent response, but CCE5 is not BAK1}

The background line for the cce5 mutants carries the so-called HVA1 transgene (in the Arabidopsis thaliana C24 ecotype), where the aequorin reporter is targeted to the tonoplast outer surface, which detects calcium exiting the vacuole but does not permit determination of absolute calcium concentrations [28]. In order to perform calibrations required for calculating absolute calcium concentrations, and to also confirm the effect of the cces mutation on cytosolic calcium levels, the five cce5 alleles were crossed into a HVA1 line (HVA1-P) that was additionally transformed with a cytosolic apoaequorin (pMAQ2) construct. These back-crossed lines also reduce possible effects from secondary mutations arising from the chemical mutagenesis. Seedlings from the F2 populations were screened for the reduced MAMP-induced calcium phenotype to identify homozygous cces plants and the 

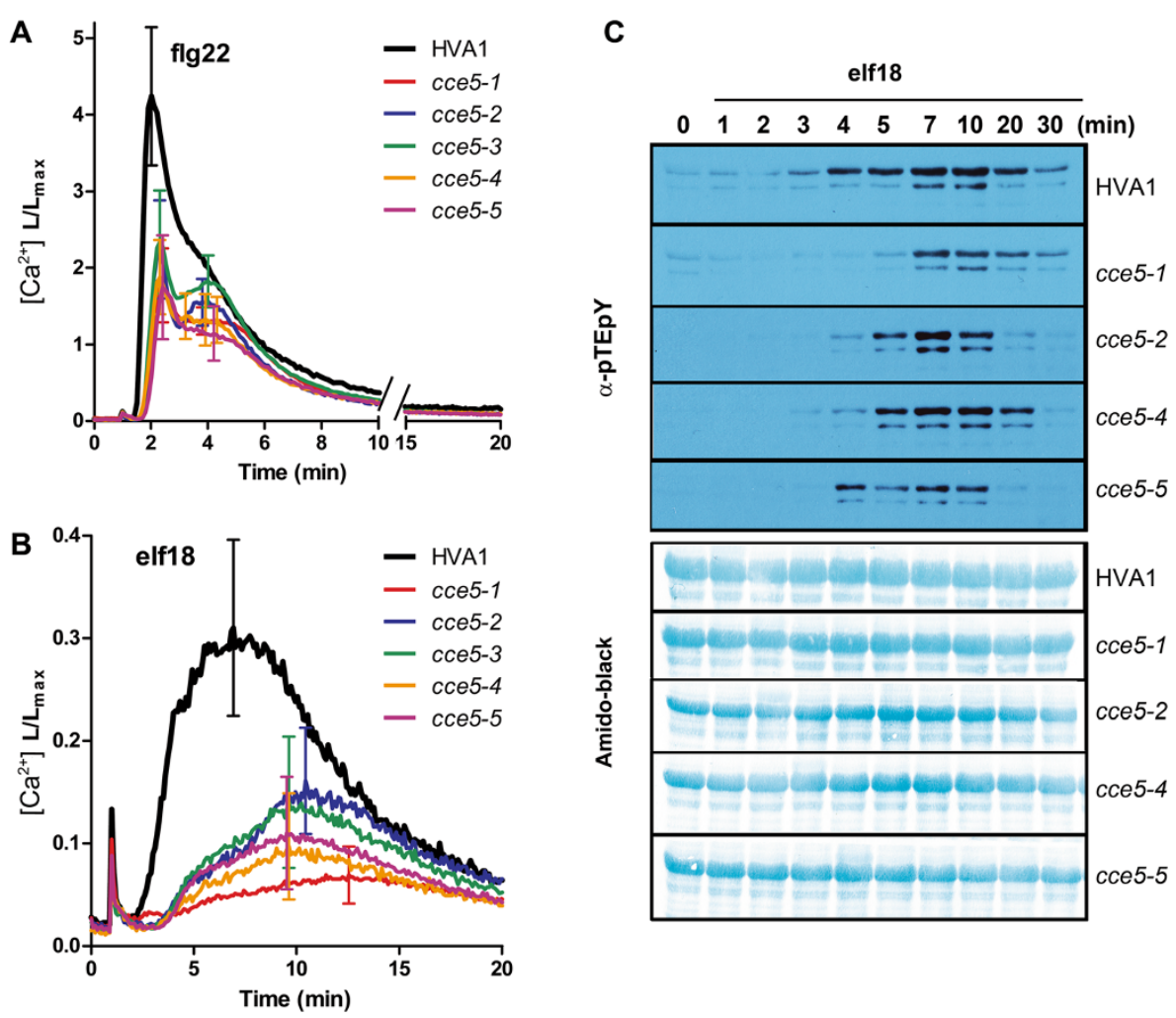

Figure 1 Early responses are reduced in the changed calcium elevation 5 (cce5) mutants compared to the parental HVA1 line. Seedlings ( 8 days old) were elicited with $1 \mu \mathrm{M}$ of flg22 (A) or $1 \mu \mathrm{M}$ elf18 (B) and the calcium levels measured. Relative calcium levels are depicted as L/Lmax ratio (where $L /$ Lmax = luminescence counts per sec/total luminescence counts remaining). Error bars represent standard deviation ( $n>12$ seedlings). MAPK activation is shown by immunoblotting (a-pTEpY) for the phosphorylated MAPKs after $1 \mu \mathrm{M}$ elf18 elicitation (C). Amido black staining of the nitrocellulose membrane was used to estimate equal loading. For MAPK activation after flg22 treatment, see Additional file 1: Figure S1.

mutations were verified by CAPS marker analysis (see Additional file 1: Table S2). Using these lines, a survey of different MAMPs/DAMPs showed reduced calcium responses to flg22, elf18 and AtPep1 but a normal response to chitin octamers (ch8) in cce5 (Figure 2). This differential phenotype to various MAMP/DAMPs is reminiscent of a BAK1-dependent type of response, where BAK1 is not required for the calcium elevation induced with ch8 [9].

BAK1 is the BRI1-associated receptor kinase shown to interact with FLS2, EFR and PEPR1/R2 receptors in a ligand-dependent manner [29]. To exclude that the cce5 mutants are weak alleles of $B A K 1$ or the related SOMATIC EMBRYOGENESIS RELATED KINASE (SERK) members, cce5-1 was crossed to the mutants bak1-4, serk4-1 and serk5-1 [5]. Since the cce5 effect was most prominent for elf18 elicitation, we measured elf18-induced calcium fluxes in F1 seedlings, and observed that the cce5 phenotype was complemented (Figure 3). This result indicates that CCE5 is not allelic to BAK1, SERK4 or SERK5 and hence cce5 is mutated in a different gene.
CCE5 encodes the receptor-like kinase, PBL1

To identify the CCE5 gene, an F2 population was generated by crossing cce5-1 with the Arabidopsis accession Ler-0. Segregation analysis with 36 F2 plants indicated that CCE5 is linked to the aequorin transgene, and located on chromosome 3 between the INDEL markers CER460928 (1 recombinant) and 473892 (1 recombinant) [30]. The map positions of CER460928 and 473892 are 17.243303 and 21.186345 Mbp (based on TAIR 10). This interval comprises 1107 gene loci, including the PBS1-like 1 gene (PBL1, At3g55450) that encodes a receptor-like cytoplasmic kinase (RLCK). Sequencing of the $P B L 1$ gene of the cce 5 mutants revealed single nucleotide polymorphisms (SNP) in all five cce5 alleles, but not in the $P B L 1$ sequences from two other cce mutants, cce7 and cce8 [26]. These SNPs lead to two premature stops (cce5-2/R110- and cce5-4/Q272-) and three amino acid exchanges (cce5-1/G70D, cce5-3/A97V and cce5-5/ $\mathrm{R} 172 \mathrm{Q})$ in the PBL1 sequence (Figure 4). Two gene models are predicted for $P B L 1$ transcripts in the TAIR 


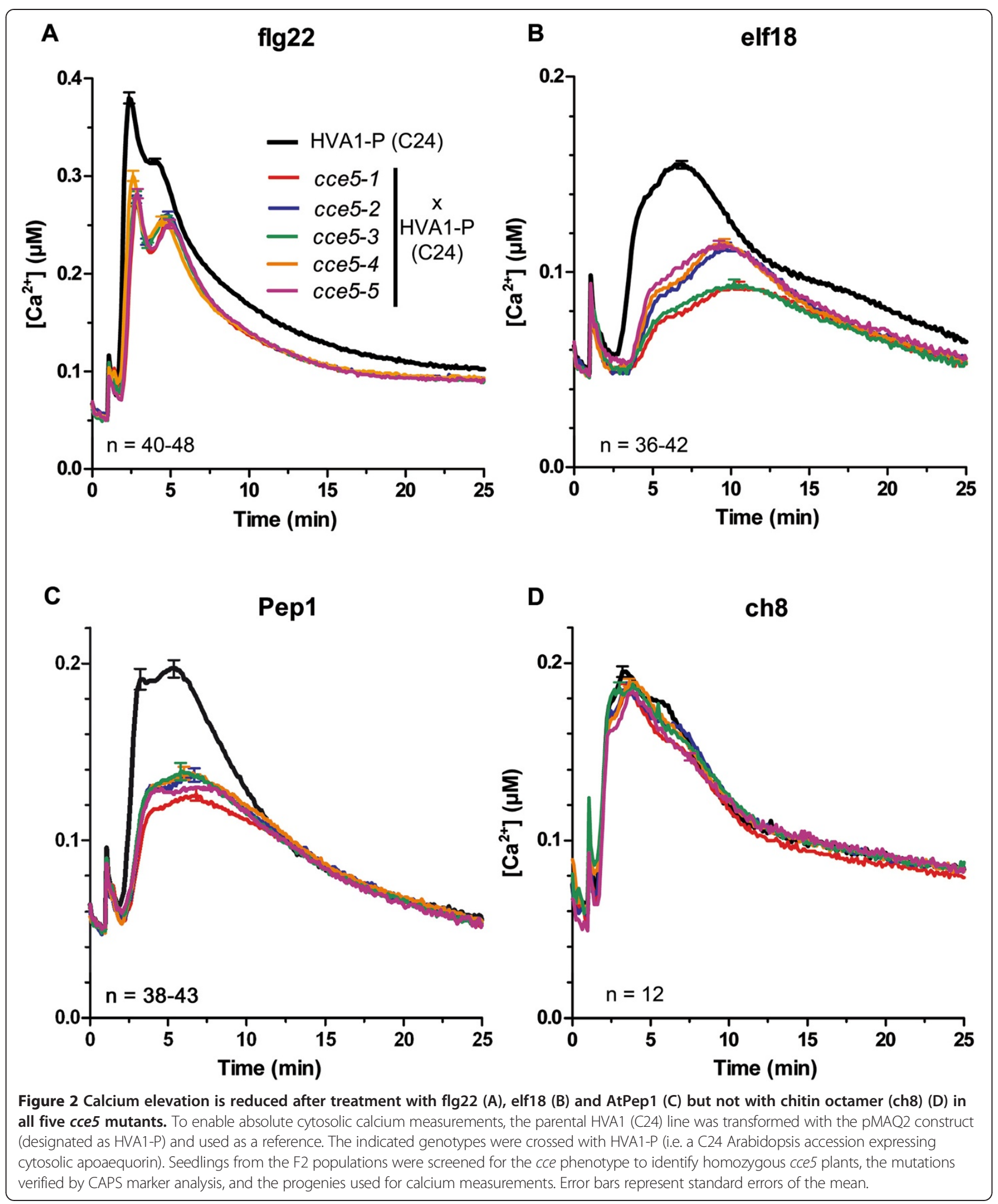

database, but since we could not detect transcripts for the predicted alternatively spliced gene model At3g55450.2 (data not shown), we used the 389 amino acid long PBL1 protein (predicted by the gene model At3g55450.1) to designate the positions of the amino acid exchanges in the cce5 mutated proteins.

PBL1 or PBS1-like 1 belongs to subfamily VII of RLCKs (Additional file 1: Figure S3) that include the founding 

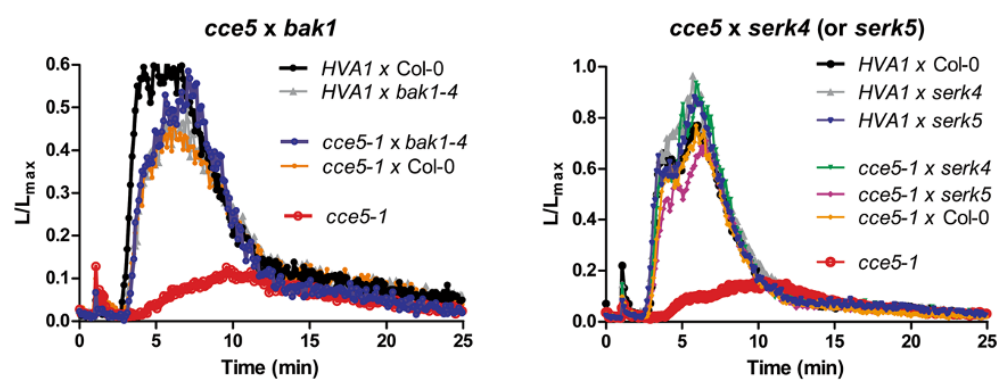

Figure 3 CCE5 is not allelic to BAK1, SERK4 or SERK5. The cce5-1 allele was crossed to bak1-4 (left panel) or to serk4 or serk5 mutants (right panel). Crosses with Col-0 or the HVA1 parent were used as controls. Relative calcium (L/Lmax) levels were determined in the F1 crosses after elf18 $(1 \mu \mathrm{M})$ treatment showing that the cce5 phenotype was complemented when crossed with the bak1, serk4 and serk5 mutants and therefore not allelic to these genes. ( $n=814$ seedlings)

member avrPphB-susceptible 1 (PBS1) [31] and the Botrytis-induced kinase 1 (BIK1) [32,33]. To further validate that CCE5 is PBL1, a T-DNA insertion mutant of PBL1 was isolated. For comparison, T-DNA mutants of related members of this family of RLCKs shown to be involved in PTI (BIK1, PBS1, PBL2) [34], were also obtained. The TDNA mutants were crossed with the cytosolic aequorinexpressing (pMAQ2 in Col-0 background) transgenic line. However, silencing of the aequorin reporter was observed in some crosses, and in these cases (i.e. for $p b l 2$ and $p b s 1$ ), an independently generated line with the apoaequorin expression driven by the UBIQUITIN10 promoter ( $p U B Q-$ $A E Q$ in Col-0 background) was used for crossing.

Consistent with the cces mutants, a reduced calcium elevation induced by flg22, elf18 and AtPep1 could be recapitulated in the pbl1 T-DNA mutant (Figure 5A). Similarly, a bik1 T-DNA mutant was compromised in calcium elevations induced by flg22, elf18 and AtPep1 whereas $p b l 2$ and $p b s 1$ showed no reduction in calcium elevation (Figure 5B,C). In the experiments with $p b l 2$ and $p b s 1$, a pbl1 line crossed with the $p U B Q-A E Q$ line was used as a control to demonstrate that the lack of phenotype in $p b l 2$ and pbs1 is not due to a different aequorin reporter

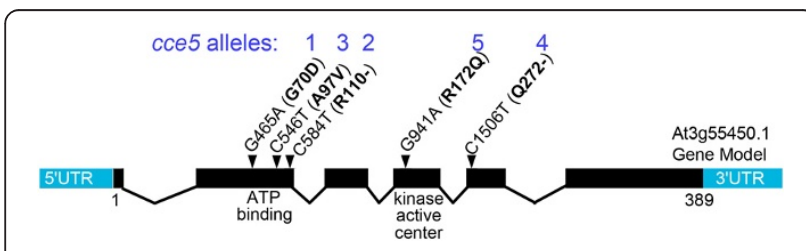

Figure 4 Scheme of PBL1 (At3g55450) gene structure and mutations in the cce5 alleles. Genomic DNA was prepared from the five cce5 mutants and the PBL1 gene amplified by PCR and sequenced. The detected single nucleotide polymorphisms (SNPs) and the resulting amino acid exchanges are indicated above the exons. The gene model At3955450.1 encoding a protein of 389 amino acids is used to designate the position of the amino acid exchanges. Locations of key kinase domains (such as ATP binding site and the kinase active center), relative to the corresponding mutations, are marked. background. Additionally, a pbl1bik1 double mutant showed further reduction of the flg22-, elf18- or AtPep1induced calcium elevations compared to the $p b l 1$ and bik1 single mutants (Figure 5A). One should also note that the altered calcium signature differs between the bik1 and pbl1 mutants (Figure 5A). Taken together, members of this RLCK family contribute differentially to MAMP/ DAMP-induced calcium elevation and there are partial redundancies between PBL1 and BIK1.

\section{Differential downstream responses in the $p b / 1$ and/or bik1 mutants}

Due to the possible trade-offs between defense and growth regulation, continuous activation of defense responses is often detrimental for plant growth. Growth inhibition assays are thus a facile measure of defense activation. This is performed by comparing root lengths of seedlings grown on normal and MAMP-containing agar plates. For this assay, we grew the two genotypes to be compared side-byside on the same plate to eliminate differences that may arise between plates (e.g. the amount of agar per plate affects the absolute amount of MAMPs available to the seedlings). Two-way-ANOVA was used to determine the statistical significance of differences in root lengths between the genotypes and the treatments, respectively. For simplicity, percent inhibition (as compared to the average root length of plants grown on standard plates) is shown in Figure 6. To reduce the effects of secondary mutations, all cce5 mutants were backcrossed to the HVA1 parent, screened for the cce phenotype and confirmed by CAPS marker analysis before the assay. Reduced flg22-mediated growth inhibition compared to the corresponding background lines could be seen for all five backcrossed cce5 mutants (Figure 6A) and the pbl1 T-DNA mutant, but not for $p b s 1$ and $p b l 2$ (Figure 6B). Surprisingly, despite the reduced calcium increase (Figure 5A), the bik1 mutant showed no reduction in root growth inhibition. There was also no additive growth reduction in the pbl1bikldouble mutant (Figure 6B). A direct comparison between the 


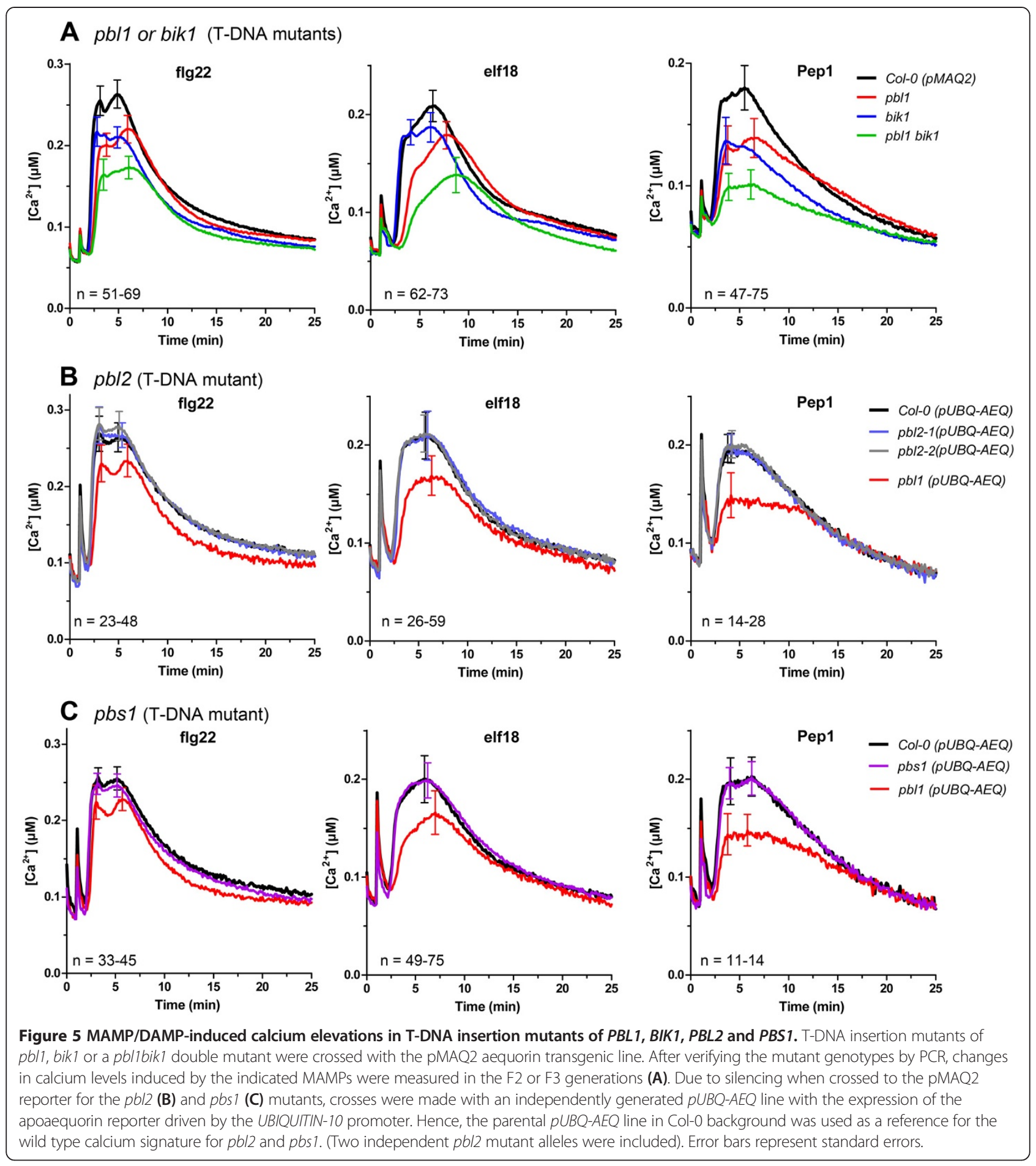

pbl1 single mutant and the pbl1bik1double mutant assayed on the same plate also showed no statistically significant difference in root growth inhibition (Figure 6B). Since $P B L 1$ and $B I K 1$ expression levels in roots are quite similar (i.e. similar signal intensities throughout the currently available microarray experiments, as analyzed by Genevestigator), the differential impact on flg22-mediated growth reduction is not due to lack of $B I K 1$ expression in roots. Thus, while $P B L 1$ and $B I K 1$ have an impact on early signaling events like calcium increase, $P B L 1$ plays a more important role than $B I K 1$ in the late root growth inhibition response to flg22. On the other hand, BIK1, but not PBL1, has been shown to play an important role in flg22mediated resistance to subsequent Pseudomonas syringae 


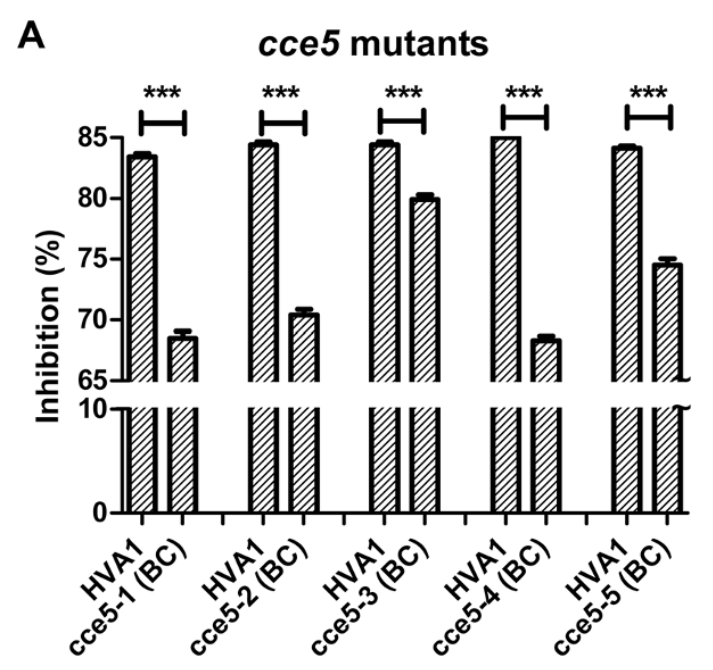

B

T-DNA mutants

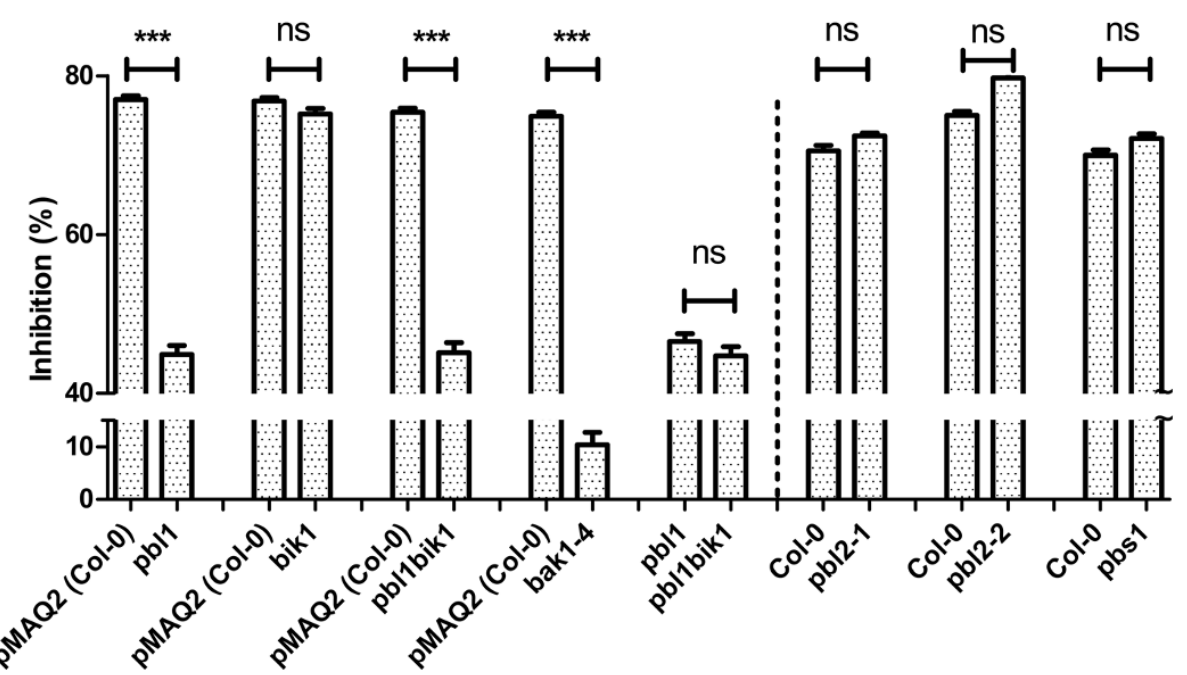

Figure 6 MAMP-induced growth inhibition is dependent on PBL1. All five cce5 alleles were backcrossed (BC) with the HVA1 parent before the growth inhibition assay (A). For the T-DNA mutants (B), either Col-0 or the PMAQ2 transgenic line (in Col-0 background) was used as reference. Wild type and mutant seedlings were grown on ATS plates with or without $1 \mu \mathrm{M}$ of flg22. Root length was measured after 14 days and depicted as percent inhibition. Two-way ANOVA was used to assess significant differences in root length (*** $=p<0.001$; ns $=$ not significant).

infection, while both BIK1 and PBL1 regulate callose deposition induced by selected MAMPs and defense gene expression [34]. Hence, PBL1 and BIK1 have overlapping but also distinct roles in defense signaling/responses as is also reflected by the wildtype-like phenotype of pbl1 plants compared to the altered growth phenotype and the constitutive SA accumulation of bik1 mutants [33].

Since calcium acts upstream of MAPK activation [9,22], we analyzed MAPK activation in the T-DNA mutants of $P B L 1, B I K 1$ and the double mutant. However, there was no reduction in MAPK activation by flg22, elf18 and Pep1 in pbl1, bik1 and pbl1bik1 compared to their Col-0 (pMAQ2) background line (Figure 7). This is in contrast to the reduced elf18-induced MAPK activation (Figure 1C) and the dose dependent reduction in flg22-induced MAPK activation (Additional file 1: Figure S1) in the cce5 mutants. Since the reduction of MAPK activation could be seen in multiple cce5 lines, the difference is unlikely to be due to secondary mutations in the ems-mutagenized lines.

Kinase activities and proper localization of RLCKs determine downstream signaling

After MAMP stimulation of plants, a reduced mobility of PBL1 and BIK1 protein bands in polyacrylamide gels (i.e. a mobility shift ), indicative of in vivo phosphorylation of the kinases, has been reported [34,35]. Since 


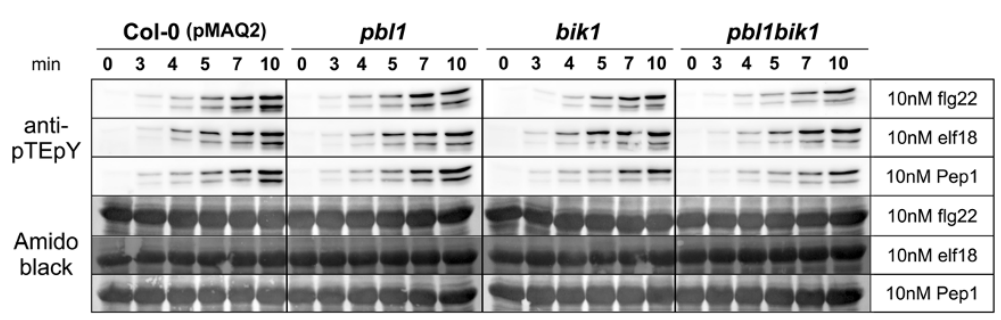

Figure 7 MAPK activation profile in the T-DNA insertion pbl1, bik1 or pbl1bik1 double mutants. 14-day-old liquid-grown seedlings were equilibrated in $1.5 \mathrm{ml}$ of fresh MS medium for $\sim 24 \mathrm{~h}$, and elicited by adding $0.5 \mathrm{ml}$ of media containing a 4-fold concentrated stock of the indicated MAMP/DAMPs. Samples were collected at the indicated time points ( $\mathrm{min}$ ) after treatment and proteins were extracted for immunoblotting to detect phosphorylated (i.e. activated) forms of the MAPKs. Amido black staining of the nitrocellulose membranes was used to estimate equal loading.

three of the cce5 alleles are predicted to encode PBL1 proteins with a single amino acid exchange (Figure 4), we tested these mutated PBL1 proteins as well as BIK1 with regard to the gel mobility shift. As a negative control, we mutated the presumed myristoylation site (G2A) of PBL1 and BIK1, which is expected to prevent the proteins from targeting to the plasma membrane. All these constructs were tagged with a C-terminal HA epitope for western blot detection and transiently expressed in Arabidopsis mesophyll protoplasts. A mobility shift could be seen for wild type PBL1 and BIK1 after flg22 treatment of the protoplasts, but not for the G2A myristoylation site variants and the G70D, A97V and R172Q PBL1 variants (Figure 8A, $\mathrm{B})$. This indicates that there is no in vivo phosphorylation of the mutated protein variants after flg22 treatment.
To test if the kinase activities have been affected, we immunoprecipitated the proteins with anti-HA antibodies and incubated the immunoprecipitates in the presence of radioactive ATP to enable autophosphorylation. After separation on a SDS-polyacrylamide gel, radioactive signals corresponding to the proteins could be seen for the wild type and the G2A mutated PBL1 and BIK1, suggesting that these are still active kinases (Figure 8C). Notably, the wild type BIK1 or PBL1 autophosphorylation signals are weak before flg22 treatment (highlighted with asterisks in Figure 8C, left panel). However, compared to the wild type PBL1 protein, there was no (or strongly reduced) autophosphosphorylation of the G70D, A97V or R172Q mutated PBL1 variants (Figure 8C). Taken together, these three cce5 mutations led to the loss of PBL1 kinase activity, while the mis-localization
A

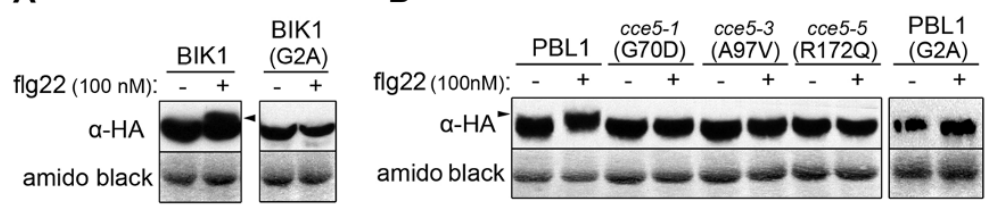

C

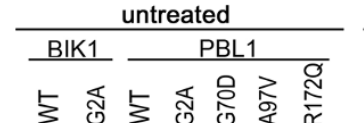

autorad.

a-HA

amido black
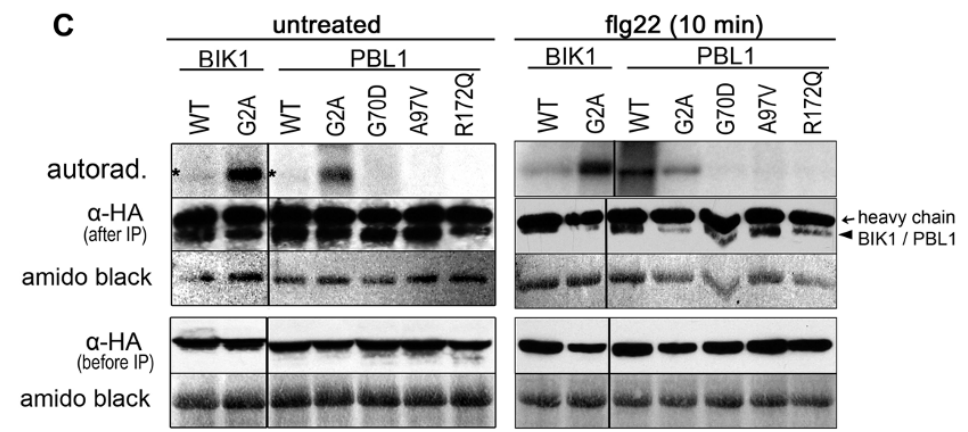

Figure 8 In vivo phosphorylation of PBL1 or BIK1 after flg22 treatment. Protoplasts were transfected with plasmids expressing HA-tagged BIK1 (A), PBL1 (B), or the indicated variants. After overnight expression of the proteins, the protoplasts were treated with $100 \mathrm{nM}$ flg22 (10 min), harvested and subjected to western blotting with anti-HA. In vivo phosphorylation is implicated by a reduced mobility of the protein (highlighted with black arrowheads). Amido black staining of the nitrocellulose membranes was used to estimate equal loading. In (C), autophosphorylation of the immunoprecipitated kinases was used to determine if the kinase activities have been compromised by the mutations. The experiment was performed three times with similar outcome. Note that the autophosphorylation of the wild type (WT) kinases in the untreated protoplasts is variable and typically low but a weak band can be seen (indicated by asterisks). Autophosphorylation of the G70D, A97V and R172Q variants were always not visible (or lower than the wild type kinases). 
of $\mathrm{PBL} 1^{\mathrm{G} 2 \mathrm{~A}}$ and $\mathrm{BIK} 1^{\mathrm{G} 2 \mathrm{~A}}$ proteins prevented the in vivo phosphorylation of these kinases after flg22 signaling.

As a final proof that PBL1 is required for the MAMPinduced calcium elevation, we introduced a genomic DNA fragment encompassing the $P B L 1$ gene locus into the pbl1 T-DNA mutant. This genomic fragment complemented the reduced calcium elevation (Figure 9A) and the root growth inhibition (Figure 9B) phenotype in pbl1. As a negative control, mutation of the putative myristoylation site (G2A) prevented the complementation of the cce phenotype (Figure 9A) as well as the flg22-induced root growth inhibition (Figure 9C). Hence, myristoylation and proper targeting of PBL1 to plasma membrane is essential for signaling function of PBL1.

\section{Discussion}

\section{Specific RLCKs required for calcium signaling and MAMP/ DAMP signaling}

Using both forward and reverse genetics as well as complementation studies, we identified the CCE5 gene as encoding the receptor-like cytoplasmic kinase, PBL1, and with the cces mutants, we isolated five new pbl1 alleles. We further showed that another RLCK, BIK1, but not PBS1 and PBL2, is required for the MAMP/DAMPinduced calcium elevation pathway. This is in agreement with a recent report that flg22-induced calcium flux is compromised in bik1 [36]. Using these new pbl1 alleles and T-DNA insertion mutants, a MAMP-mediated root growth inhibition assay confirmed the requirement of PBL1 for downstream signaling leading to growth arrest. However, the bik1 mutation had no apparent effect on flg22-mediated growth arrest. Thus, despite both pbl1 and bik1 mutants showing a reduced MAMP/DAMPinduced calcium elevation, downstream growth arrest effects differ. One explanation is that PBL1 and BIK1 are not simply redundant but also have distinct signaling roles, which is reinforced by other studies showing both overlapping and distinct requirements for PBL1 and BIK1 in MAMP-induced ROS production, callose deposition, gene expression and pathogen resistance $[33,34,37,38]$. This notion is, in fact, evident from the different calcium signatures in the pbl1 and bik1 mutants (see Figure 5A). A second possibility is that a signaling critical threshold of cytoplasmic calcium level is not crucial or causal for determining the degree of growth inhibition (or other MAMP-induced responses), which would imply a more important signaling role of PBL1, compared to BIK1, in mediating the possible trade-offs between growth and defense activation. It is possible that the inverse roles of BIK1 as a positive regulator of defense but a negative regulator of brassinosteroid signaling [39] contribute to this growth differences. Recently, another RLCK, PBL27, was found to be the preferential substrate (as compared to BIK1) of the CERK1 chitin receptor. By contrast, BAK1, which is already known to phosphorylate BIK1, hardly phosphorylated PBL27. PBL27 also appears to be nonessential for flg22 signaling [40]. Thus, depending on the ligand, different RLKs or RLCKs are recruited for signaling. These findings support the distinction of BAK1 requirement for flg22, elf18 and AtPep1 signaling to that of chitin [5,9]. Our data on PBL1 requirement for optimal calcium signaling induced by flg22, elf18 and AtPep1, but not chitin (Figure 5), fits into this pattern. In conclusion, there appears to be a differential requirement for members of the RLCK family downstream of the receptors for distinct MAMP/DAMP signaling.

\section{Phosphorylation is essential for signal relay}

The recruitment (and/or exchange) of various RLKs and RLCKs at the plasma membrane after MAMP/DAMP perception is indicative of the roles of phosphorylation cascades in early signaling. Prior to stimulation, FLS2 and BIK1 are already in a protein complex [38] and BAK1 appears to be also in complex with BAK1interacting RLKs (BIRs) [41]. Within minutes after flg22 stimulation, FLS2 recruits BAK1 [3] to phosphorylate BIK1. Activated BIK1, in turn, cross-phosphorylates FLS2 and BAK1 [38]. BAK1 also cross-phosphorylates FLS2 but apparently at different residues as BIK1 [42]. Based on the autophosphorylation assay (Figure 8C), the kinase activities of BIK1 and PBL1 appear to be higher in the flg22-treated protoplasts. The lower activities of BIK1/PBL1 in the untreated protoplasts may imply repression by some other components (eg. phosphatases) prior to elicitation. Along this idea, it is noteworthy that the N-terminal myristoylation $\mathrm{BIK} 1^{\mathrm{G} 2 \mathrm{~A}}$ and $\mathrm{PBL} 1^{\mathrm{G} 2 \mathrm{~A}}$ mutants are routinely recovered with higher autophosphorylation levels. One may speculate that mislocalization of PBL1 ${ }^{\mathrm{G} 2 \mathrm{~A}}$ and $\mathrm{BIK} 1^{\mathrm{G} 2 \mathrm{~A}}$ prevent contact with phosphatases that are presumably present in the FLS2-BIK1 (or PBL1) protein complex to restrict defense signaling. Indeed, Ser/Thr protein phosphatase type 2A (PP2A) has been shown to associate with BAK1 and control the activation of PRR complexes [43]; and whether the same or similar PP2As negatively regulate BIK1 or PBL1 remains to be demonstrated. Recently, it was shown that both BAK1 and BIK1 are dual-specific kinases that modify both serine/threonine as well as tyrosine residues [44]. The complex series of phosphorylation between PRRs, BAK1, BIK1 and PBL1 are important as mutations abrogating activities of any of these kinases block signaling. As shown by mobility shifts in gel electrophoresis, the PBL1 protein, encoded by the CCE5 gene, is apparently phosphorylated in vivo after MAMP elicitation [34]. The loss (or reduction) of the kinase activities of the cce5-derived protein variants reported here (Figure 8C) corresponds to changes of 


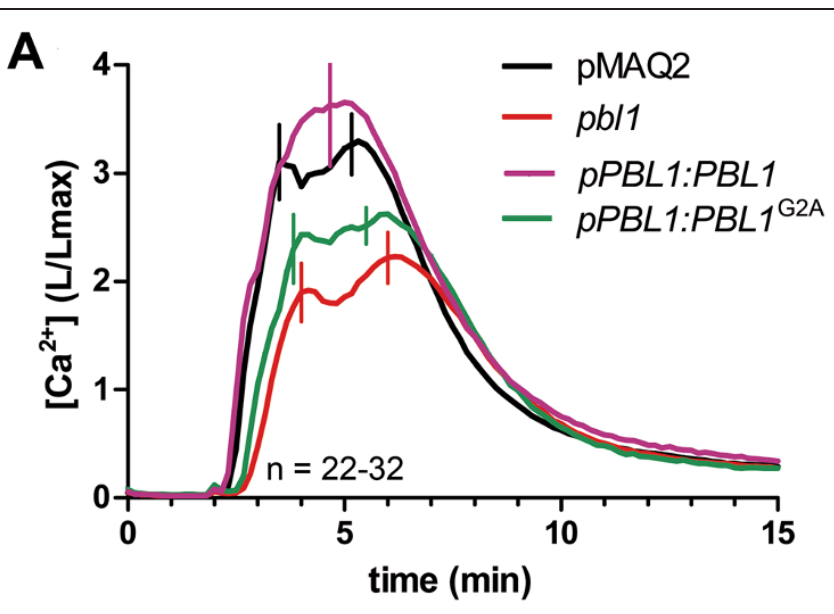

B Complementation with PBL1
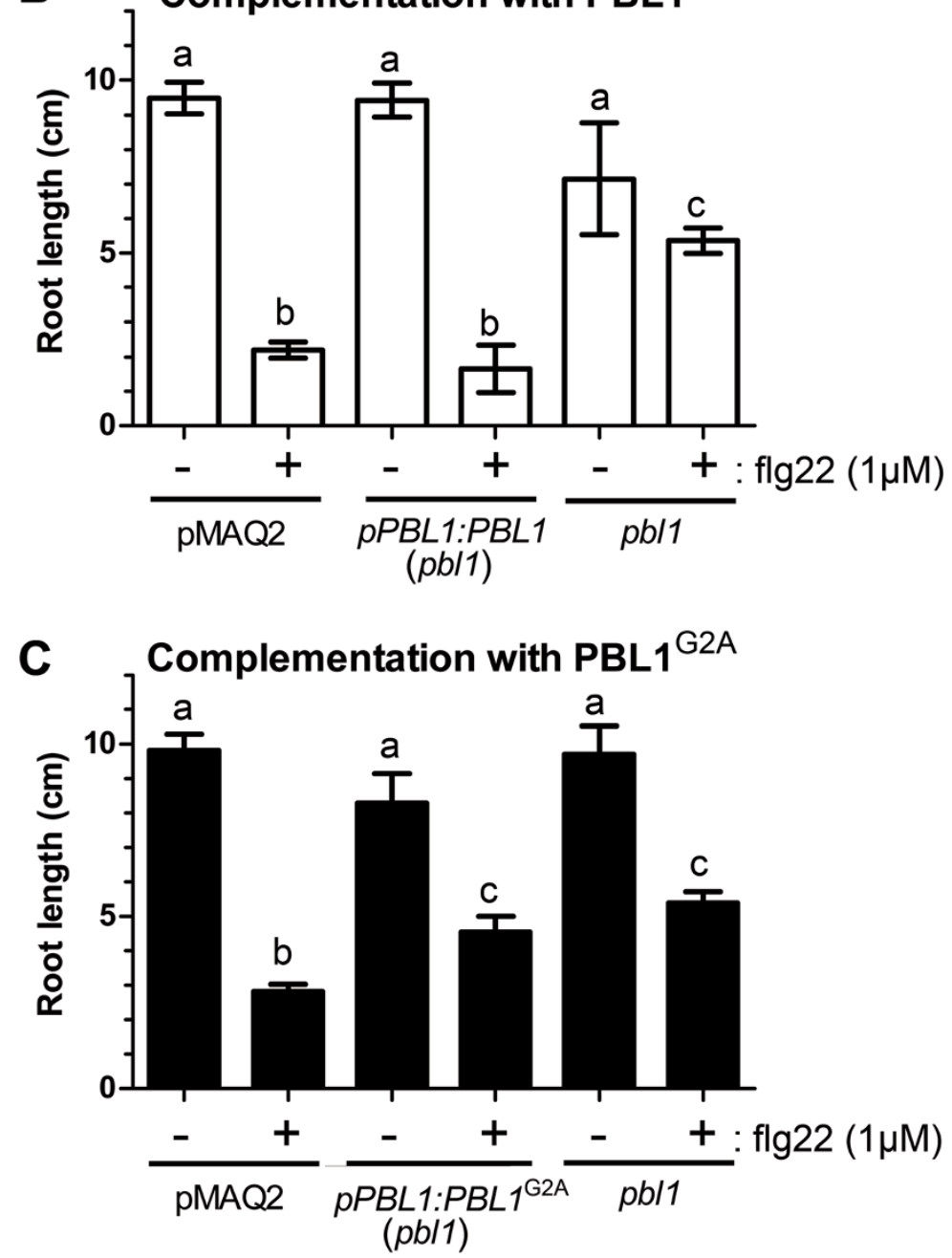

Figure 9 Complementation of pbl1 T-DNA mutant. The T-DNA pbl1 mutant was transformed with a genomic fragment encompassing the PBL1 locus. As a negative control, mutation of the myristoylation site (G2A) prevented complementation in the flg22-induced calcium elevation phenotype (A) and the flg22-mediated growth arrest (B, C). In (A), the calcium levels of the PPBL 1:PBL ${ }^{G 2 A}$-complemented seedlings (green trace) are not statistically significant from calcium levels in the pbl1 mutant (red trace). In (B) and (C), the letters above each bar show the statistical significance groupings based on two-way ANOVA. ( $n=1427$ seedlings). 
important residues of the kinase domain. The G70D and A97V mutations are found in the ATP binding region (i.e. the kinase subdomains I and II, respectively) while R172Q is N-terminal to the active center within kinase subdomain VI (c.f. Figure 4 and Figure 8C). Taken together with data from literature, the reduced calcium signaling of our newly isolated cce5/pbl1 alleles shows that kinase activities of PBL1 (and all the other recruited RLKS/RLCKs) are vital for early MAMP/DAMP signaling.

\section{Is downstream MAPK activation affected in the pbl1 and bik1 mutants?}

Downstream of PBL1/BIK1 phosphorylation is calcium elevation, which, in turn, has been shown through pharmacological inhibitor studies to be required for downstream MAPK activation [22]. However, despite an attenuation of calcium elevation, there was no reduction in MAPK activation by flg22, elf18 or AtPep1 in the pbl1 and bik1 T-DNA mutants as well as pbl1bik1 double mutant when compared to their Col-0 (pMAQ2) background line (Figure 7). This observation is in agreement with previous reports $[34,45,46]$. On the other hand, we observed a reduction/delay in elf18induced MAPK activation in the cce5 mutants (Figure 1C), while for flg22, a weak effect could be observed when a lower concentration of the flg22 peptide was used. A possible explanation for this discrepancy may be that the mutated or truncated CCE5 proteins (with inhibitory properties) are expressed by the cce 5 alleles as opposed to the (presumably) lack of proteins in the T-DNA insertion mutants. Alternatively, the different genetic background of the mutants may also play a role. In all studies where no difference in MAPK activation was observed, the mutations were in Col-0, while the cce5 (pbl1) mutants have a C24 background. We previously reported that the $\mathrm{C} 24$ accession has higher levels of FLS2 receptor while, on the basis of public gene expression profiling data, the opposite is true for the EFR receptor [26]. Several SNPs were also detected within the FLS2 and EFR genes of HVA1 (C24) [26], which may further contribute to the different sensitivities to flg22 and elf18. Together, this may explain the observed reduced elf18-induced MAPK activation in the cce5 mutants but only a dose-dependent flg22-induced MAPK response. Along this notion, Zhang et al. [34] also reported that callose deposition in the pbl1 mutant was normal upon flg22 treatment but reduced when treated with elf18 [34]. Thus, the differential MAMP receptor levels and/or yet unknown alterations in other signaling components between genotypes may determine the sensitivity of the system in signal relay from PBL1 to the MAPKs and other downstream events. Additionally, the RLCK, RIPK, phosphorylates RIN4 and in analogy to the recognition of the RIN4 phosphorylation by the RPM1 resistance protein as compared to recognition of RIN4 cleavage by RPS2 [47], one could speculate that the MAMP-induced RLCK phosphorylation may also be differentially recognized by the differing configuration of resistance protein spectrum between Arabidopsis accessions.

\section{Localization to the membrane is a prerequisite for PBL1 and BIK1 function}

Besides its kinase activities, localization of PBL1 and BIK1 appears to be important for complementation of the cce phenotype. BIK1, as a GFP fusion protein, has been shown to be plasma membrane-localized using heterologous expression in onion epidermal cells [33]. However, to our knowledge, it has never been experimentally determined whether this is due to targeting or recruitment by other proteins. CASTAWAY, an RLCK required for organ abscission, showed reduced plasma membrane localization when a G2A mutation was introduced for the putative myristoylation site. Myristoylation is often associated with palmitoylation to enhance membrane interactions. However, no further decrease of CASTAWAY localization was observed even when the neighboring palmitoylation site (C4S) was additionally mutated [48]. Myristoylation is thought to provide the initial but weak interaction with the plasma membrane; stabilization of this membrane localization may be further strengthened through other modifications or interactions with resident plasma membrane-localized components [49]. We now show that the G2A mutation of the putative myristoylation site of PBL1/BIK1 prevented signaling (i.e. no in vivo phosphorylation after MAMP treatment, Figure 8) although kinase (autophosphorylation) function is apparently intact. Furthermore, the G2A variant did not complement the pbl1 mutation (Figure 9). In a similar manner, despite being cleaved by the avrPphB cysteine protease, the active PBS1 fragment that is recognized must be retained at the plasma membrane for RPS5 activation [50]. In this case, plasma membrane targeting is mediated by $\mathrm{S}$-acylation of a cysteine residue in the $\mathrm{N}$-terminus of PBS1. Thus, not only the function of the PBS1 protein but also the recognition of its perturbation (i.e. ETI) requires correct membrane localization. Taken together, localization of the RLCKs to the proper cell compartment is crucial for function.

\section{Disabling RLCK functions during pathogenesis blocks defense signaling}

PBS1 is the founding member of the PBL (PBS1-like) group. The Pseudomonas avrPphB effector, a bacterial virulence protein injected into host cells, cleaves PBS1 via its cysteine protease activity [31]. Subsequently, it was discovered that avrPphB can cleave at least 10 other PBS1-like RLCKs [34]. Their cleavage/removal represents a virulence function of avrPphB and suggests that these PBLs/RLCKs act in resistance mechanisms against bacteria. Support for this notion is provided by studies involving the Xanthomonas XopAC effector, which appears to target multiple 
RLCKs [37]. Unlike avrPphB, XopAC does not cleave but uridylates BIK1 and RIPK in susceptible plants [45]. This transfer of uridine $5^{\prime}$-monophosphate to conserved phosphorylation sites in the activation loop of BIK1 and RIPK, prevents phosphorylation, thereby reducing their kinase activities and consequently inhibiting downstream defense signaling. In accordance to the arms-race hypothesis, XopAC appears to be a major avirulence factor for recognition in resistant plants such as the Arabidopsis Col-0 accession. Furthermore, there is more growth of Xanthomonas campestris pv. campestris expressing XopAC in the pbl2 background. This suggests that the RLCK, PBL2 is required for the XopAC-triggered immunity [37]. These observations of various pathogen effectors targeting RLCKs are in line with the presumed importance of RLCKs in defense signaling. However, although avrPphB cleaves multiple PBLs/RLCKs [34], only PBS1 cleavage is recognized by RPS5 [27,51]. We report here that PBL1 and BIK1, but neither PBL2 nor PBS1, are required for MAMP/DAMP-induced calcium signaling (Figure 5). This raises the question of why avrPphB would target PBS1 if PBS1 is not important for MAMP-induced calcium signaling. In fact, there is no evidence so far for any importance of PBS1 in pathogen resistance. As proposed by Zhang et al. [46], one idea is that PBS1 may be a decoy [52] evolved to recognize perturbation of the real targets of the pathogen effectors. In this case, PBL1 and BIK1 would be such real avrPphB targets, which are relevant for cross-phosphorylation of the receptor complex components and the subsequent triggering of calcium and downstream defense signaling.

\section{Conclusion}

In summary, we showed the requirement for the two RLCKs, PBL1 and BIK1, in MAMP/DAMP-induced calcium signaling, and speculated on possible genotype variations that may differentially contribute to downstream signaling events. There are many more RLCK genes in the genome (Additional file 1: Figure S3) and it remains to be investigated whether these also affect early calcium signal transduction perhaps in dependence on the type of MAMP/DAMP ligands. The large number of available RLCKs is presumably mirrored by an even wider repertoire of their downstream substrates. Besides phosphorylating receptor complex components, BIK1 was recently shown to target the NADPH oxidase, RBOHD, to control oxidative burst in a calcium-independent manner [36,53]. Thus, a future challenge would be to identify the substrates of these various RLCKs and elucidate their role in cellular signaling.

\section{Methods}

Plant lines and cultivation conditions

The Arabidopsis thaliana lines pMAQ2 in Col-0 background and HVA1 in C24 background were obtained from $M$. and $H$. Knight [25]. These lines express the apoaequorin gene under the control of the cauliflower mosaic virus $35 \mathrm{~S}$ promoter. In the case of HVA1, the aequorin is targeted as a pyrophosphatase $(\mathrm{H}+-\mathrm{PPase})$-apoaequorin fusion protein to the cytoplasmic-face of the tonoplast in the so-called vacuolar microdomain (vmd); thus enabling measurements of calcium changes at this vacuolar vicinity. For the backcross shown in Figure 2, the HVA1 (C24) line was first retransformed with pMAQ2 construct to obtain a line expressing additionally cytosolic apoaequorin (designated HVA1-P). This HVA1-P line was then crossed to the cce5 mutants. T-DNA lines used in this study are listed in the Additional file 1: Table S1). Plants for ROS assays were grown on soil in climate chambers under short day conditions (8 h light, $16 \mathrm{~h}$ dark cycles). For calcium and MAPK assays, seeds were surface-sterilized, stratified at 4 ? C for $>2$ $\mathrm{d}$ and grown in liquid MS under long day conditions $(16 \mathrm{~h}$ light, $8 \mathrm{~h}$ dark cycles) as described [25].

\section{Calcium measurements}

Seed sterilization, growth of seedlings and other experimental set-up for the calcium measurements in a 96well plate format was performed as described [26].

\section{ROS, MAPK and growth inhibition assays}

Detection of early MAMP-triggered responses such as MAPK activation and reactive oxygen species (ROS) accumulation was performed as described [9]. As a late response to MAMPs, growth inhibition assay was performed as described [26]. Briefly, seedlings were grown vertically on ATS agar plates with or without $1 \mu \mathrm{M}$ flg22 for 14 days. To distinguish between growth differences due to treatment versus genotype effects, two-way ANOVA was performed on $\log _{2^{-}}$ transformed root length data (genotype vs. treatment; $\mathrm{p}<$ 0.001; R statistical package) [54]. For a more compact and simplified overview, data in Figure 6 were depicted as percent growth inhibition compared to control.

\section{Transient expression in protoplasts, immunoprecipitation and autophosphorylation}

Transient expression in Arabidopsis protoplasts was performed as described [9]. For each sample, $1 \mathrm{ml}$ of protoplasts $\left(\sim 2 \times 10^{5}\right.$ protoplasts $\left.\mathrm{ml}^{-1}\right)$ was transformed; of which $300 \mu \mathrm{l}$ were kept for western blot analysis of protein expression. The remaining $700 \mu \mathrm{l}$ were used for immunoprecipitation. Proteins were extracted from the transfected protoplasts as described except that the extraction buffer was supplemented with $1 \%$ Triton X-100 [55]. The proteins were incubated with anti-HA (Covance) and protein-G-sepharose (for at least $2 \mathrm{~h}, 4$ ? C). Washing of the beads was performed as in Lee et al. [55], with centrifugation in between washes to pellet the sepharose beads. Finally, sepharose beads with the immunoprecipitated proteins were resuspended in $20 \mu \mathrm{l}$ of kinase buffer (20 mM Hepes $\mathrm{pH} 7.5,15 \mathrm{mM} \mathrm{MgCl}$, 
$5 \mathrm{mM}$ EGTA, $1 \mathrm{mM}$ DTT); $5 \mu \mathrm{l}$ was kept for a western blot to confirm recovery of the HA-tagged proteins. $\gamma^{32}$ P-ATP $\left(3000 \mathrm{Ci} \mathrm{mmol}^{-1}\right)(0.1 \mu \mathrm{l})$ was added to the remaining $15 \mu \mathrm{l}$ and incubated at 30 ? C for $1 \mathrm{~h}$ to initiate autophosphorylation. Five $\mu \mathrm{l}$ of $4 \mathrm{xSDS}$-loading buffer was added to the beads, incubated at 95? C for $5 \mathrm{~min}$, and $12 \mu \mathrm{l}$ loaded on a 10\% SDS-PAGE. After electrophoresis, the gel was dried, exposed overnight and analyzed by Phosphorimaging.

\section{Mapping}

An F2 population was generated from the cross of $c c e 5-1$ (C24) with the Arabidopsis ecotype Landsberg erecta (Ler-0). F2 plants containing the aequorin transgene (i.e. showing coelenterazine-dependent luminescence) were selected and selfed. DNA was isolated from leaves of F2 plants or from pooled F3 seedlings, and 3 to 5 markers (SNPs, INDELs) of each chromosome were genotyped. Calcium measurements were performed with the F3 seedlings and the segregation of the phenotype used to infer if the corresponding F2 parent is heterozygous or homozygous for the cce5 mutation.

\section{Molecular cloning, plant transformation and complementation}

For complementation analysis, a genomic fragment covering the PBL1-ORF and $2 \mathrm{~kb}$ upstream cis-regulatory region was amplified by PCR using Phusion? Hot Start High-Fidelity DNA polymerase (Thermo Scientific) with primers PBL1-Prom/-STOP and cloned into pENTR / $\mathrm{D}-\mathrm{TOPO}$ according to manufacturer s instructions. $\mathrm{Mu}$ tation of the N-myristoylation site (G2A) was performed using the QuikChangeII-Kit (Stratagene) with primers PBL1-NMSmut-F/-R according to manufacturers instructions. Clones were verified by sequencing and transferred via LR reaction into destination vector pGWB1 to obtain $p P B L 1::$ PBL1 and $p P B L 1::$ PBL1(G2A). After transfer of the constructs into Agrobacterium tumefaciens (GV3101), Arabidopsis pbl1 mutant plants were transformed by floral-dip transformation. Transgenic plants were selected on hygromycin-containing plates and crossed with pbl1-AEQ to introduce the apoaequorin transgene. For transient expression in protoplasts, PBL1- and BIK1-ORFs were amplified from cDNA obtained from Col-0 or the indicated cce 5 alleles by PCR using Phusion? Hot Start High-Fidelity DNA polymerase (Thermo Scientific) with primers PBL1START/-NoSTOP and BIK1-START/-NoSTOP and cloned into $\mathrm{pENTR}{ }^{\mathrm{TM}} / \mathrm{D}-\mathrm{TOPO}$ according to manufacturer s instructions. Mutation of the N-myristoylation site (G2A) was introduced using primers PBL1STARTmut and BIK1-STARTmut. Clones were verified by sequencing and transferred via LR reaction into destination vector pUGW14 to obtain $p 35 S::$ PBL1-3xHA,
p35S::BIK1-3xHA, $p 35 s::$ PBL1(G2A)-3xHA and $p 35 S::$ BIK1(G2A)-3xHA.

The aequorin-ORF was amplified from plasmid pMAQ2 by PCR using Phusion? Hot Start High-Fidelity DNA polymerase (Thermo Scientific) with primers AEQ-START/-STOP, cloned into $\mathrm{pENTR}^{\mathrm{TM}} / \mathrm{D}-\mathrm{TOPO}$ according to manufacturer s instructions, verified by sequencing and transferred via LR reaction into destination vector $\mathrm{pUB}-\mathrm{DEST}$ to obtain $p U B Q 10: \mathrm{AEQ}$. After transfer of the construct into Agrobacterium tumefaciens (GV3101), Arabidopsis Col-0 plants were transformed by floral-dip transformation and transgenic plants were selected by spraying with BASTA? (glufosinat-ammonium; Bayer). All primers used for cloning are listed in the Additional file 1: Table S3).

\section{Availability of supporting data}

All the supporting data are available within the article or as additional files. The phylogenetic tree (Additional file 1: Figure S3) has been deposited in treebase (ID: 16757) and the data will be available at the following URL: http://purl. org/phylo/treebase/phylows/study/TB2:S16757.

\section{Additional file}

Additional file 1: Figure S1. MAPK activation in cce5 mutants. Reduced
MAPK activation in cce5-1 mutant is seen with flg22 elicitation at low
concentrations (10 nM) (A) but not obvious at higher concentrations (100
nM flg22) (B). Figure S2. MAMP-induced reactive oxygen species (ROS)
accumulation. Reduced ROS accumulation in the cce5 mutants after elf18
(A) or flg22 (B) treatments. Figure S3. Evolutionary relationships of 51
group VII RLCKs. Table S1. Mutant lines used in this study. Table S2.
CAPS markers for genotyping the cce5 mutant alleles. Table S3. Primers
used for molecular cloning.

\section{Competing interests}

The authors declare that they have no competing interests.

\section{Authors contributions}

SR designed experiments and together with KF, carried out the calcium, MAPK, genetic crosses, plant transformation, and complementation experiments. LE-L performed the protoplast transformation studies. LW assisted in genetic mapping of the cce5 mutation. DS and JL supervised and coordinated. $J$ drafted the manuscript. All authors read and approved the final manuscript.

\section{Acknowledgements}

This work is financed by the German Research Foundation, through the priority SPP1212 Plant Micro program to J.L (LE 2321/1-3) and the Collaborative Research Center program SFB648 to J.L. and D.S. L.E.-L. is supported within the ProNet-T3 program (03ISO2211B). We thank Nicole Bauer, Marina Huler and Siska Herklotz for excellent technical assistance.

\section{Author details}

${ }^{1}$ Stress and Developmental Biology, Leibniz Institute of Plant Biochemistry, Weinberg 3, Halle/Saale D-06120, Germany. ${ }^{2}$ Phytopathology, Center of Life and Food Sciences Weihenstephan, Technische Universitt Mnchen, Emil-Ramann-Str. 2, Freising, Weihenstephan D-85350, Germany.

Received: 6 August 2014 Accepted: 8 December 2014

Published online: 19 December 2014 


\section{References}

1. Boller T, Felix G: A renaissance of elicitors: perception of microbeassociated molecular patterns and danger signals by pattern-recognition receptors. Annu Rev Plant Biol 2009, 60:379 406.

2. Monaghan J, Zipfel C: Plant pattern recognition receptor complexes at the plasma membrane. Curr Opin Plant Biol 2012, 15(4):349 357.

3. Chinchilla D, Zipfel C, Robatzek S, Kemmerling B, Nrnberger T, Jones JD, Felix G, Boller T: A flagellin-induced complex of the receptor FLS2 and BAK1 initiates plant defence. Nature 2007, 448(7152):497 500.

4. Postel S, Kufner I, Beuter C, Mazzotta S, Schwedt A, Borlotti A, Halter T, Kemmerling B, Nurnberger T: The multifunctional leucine-rich repeat receptor kinase BAK1 is implicated in Arabidopsis development and immunity. Eur J Cell Biol 2009, 89(2 3):169 174

5. Roux M, Schwessinger B, Albrecht C, Chinchilla D, Jones A, Holton N, Malinovsky FG, Tor M, de Vries S, Zipfel C: The Arabidopsis leucine-rich repeat receptor-like kinases BAK1/SERK3 and BKK1/SERK4 are required for innate immunity to hemibiotrophic and biotrophic pathogens. Plant Cell 2011, 23(6):2440 2455.

6. Wang ZY, Seto H, Fujioka S, Yoshida S, Chory J: BRI1 is a critical component of a plasma-membrane receptor for plant steroids. Nature 2001, 410(6826):380 383.

7. Sun Y, Li L, Macho AP, Han Z, Hu Z, Zipfel C, Zhou JM, Chai J: Structural basis for flg22-induced activation of the Arabidopsis FLS2-BAK1 immune complex. Science 2013, 342(6158):624 628.

8. Krol E, Mentzel T, Chinchilla D, Boller T, Felix G, Kemmerling B, Postel S, Arents M, Jeworutzki E, Al-Rasheid KA, Becker D, Hedrich R: Perception of the Arabidopsis danger signal peptide 1 involves the pattern recognition receptor AtPEPR1 and its close homologue AtPEPR2. J Biol Chem 2010, 285(18):13471 13479.

9. Ranf S, Eschen-Lippold L, Pecher P, Lee J, Scheel D: Interplay between calcium signalling and early signalling elements during defence responses to microbe- or damage-associated molecular patterns. Plant $J$ 2011, 68(1):100 113 .

10. Schwessinger B, Roux M, Kadota Y, Ntoukakis V, Sklenar J, Jones A, Zipfel C Phosphorylation-dependent differential regulation of plant growth, cell death, and innate immunity by the regulatory receptor-like kinase BAK1. PLOS Genet 2011, 7(4):e1002046.

11. Shan L, He P, Li J, Heese A, Peck SC, Nurnberger T, Martin GB, Sheen J: Bacterial effectors target the common signaling partner BAK1 to disrupt multiple MAMP receptor-signaling complexes and impede plant immunity. Cell Host Microbe 2008, 4(1):17 27.

12. Miya A, Albert P, Shinya T, Desaki Y, Ichimura K, Shirasu K, Narusaka Y, Kawakami N, Kaku H, Shibuya N: CERK1, a LysM receptor kinase, is essential for chitin elicitor signaling in Arabidopsis. Proc Natl Acad Sci U S A 2007, 104(49):19613 19618.

13. Petutschnig EK, Jones AM, Serazetdinova L, Lipka U, Lipka V: The LysM-RLK CERK1 is a major chitin binding protein in Arabidopsis thaliana and subject to chitin-induced phosphorylation. J Biol Chem 2010, 285(37):28902 28911.

14. Wan J, Zhang XC, Neece D, Ramonell KM, Clough S, Kim SY, Stacey MG, Stacey G: A LysM receptor-like kinase plays a critical role in chitin signaling and fungal resistance in Arabidopsis. Plant Cell 2008, 20(2):471 481

15. Ranf S, Wunnenberg P, Lee J, Becker D, Dunkel M, Hedrich R, Scheel D, Dietrich $\mathrm{P}$ : Loss of the vacuolar cation channel, AtTPC1, does not impair $\mathrm{Ca}^{2+}$ signals induced by abiotic and biotic stresses. Plant J 2008, 53(2):287 299.

16. Blume B, Nrnberger T, Nass N, Scheel D: Receptor-mediated increase in cytoplasmic free calcium required for activation of pathogen defense in parsley. Plant Cell 2000, 12(8):1425 1440.

17. Samaj J, Ovecka M, Hlavacka A, Lecourieux F, Meskiene I, Lichtscheidl I, Lenart P, Salaj J, Volkmann D, Bogre L, Baluska F, Hirt H: Involvement of the mitogen-activated protein kinase SIMK in regulation of root hair tip growth. Embo J 2002, 21(13):3296 3306.

18. Seybold H, Trempel F, Ranf S, Scheel D, Romeis T, Lee J: $\mathrm{Ca}^{2+}$ signalling in plant immune response: from pattern recognition receptors to $\mathrm{Ca}^{2+}$ decoding mechanisms. New Phytol 2014, 204:782 790.

19. Steinhorst $L$, Kudla J: Calcium and reactive oxygen species rule the waves of signaling. Plant Physiol 2013, 163(2):471 485.

20. Torres MA, Dangl JL, Jones JD: Arabidopsis gp91phox homologues AtrbohD and AtrbohF are required for accumulation of reactive oxygen intermediates in the plant defense response. Proc Natl Acad Sci U S A 2002, 99(1):517 522
21. Dubiella U, Seybold H, Durian G, Komander E, Lassig R, Witte CP, Schulze WX, Romeis T: Calcium-dependent protein kinase/NADPH oxidase activation circuit is required for rapid defense signal propagation. Proc Natl Acad Sci U S A 2013, 110(21):8744 8749.

22. Ligterink W, Kroj T, zurNieden U, Hirt H, Scheel D: Receptor-mediated activation of a MAP kinase in pathogen defense of plants. Science 1997 276(5321):2054 2057.

23. Aslam SN, Newman MA, Erbs G, Morrissey KL, Chinchilla D, Boller T, Jensen T, De Castro C, Lerano T, Molinaro A, Jackson RW, Knight MR, Cooper RM: Bacterial polysaccharides suppress induced innate immunity by calcium chelation. Curr Biol 2008, 18(14):1078 1083.

24. Knight $H$, Knight MR: Imaging spatial and cellular characteristics of low temperature calcium signature after cold acclimation in Arabidopsis. J Exp Bot 2000, 51(351):1679 1686.

25. Knight H, Trewavas AJ, Knight MR: Cold calcium signaling in Arabidopsis involves two cellular pools and a change in calcium signature after acclimation. Plant Cell 1996, 8(3):489 503.

26. Ranf S, Grimmer J, Pschl Y, Pecher P, Chinchilla D, Scheel D, Lee J: Defense-related calcium signaling mutants uncovered via a quantitative high-throughput screen in Arabidopsis thaliana. Mol Plant 2012, 5(1):115 130.

27. Swiderski MR, Innes RW: The Arabidopsis PBS1 resistance gene encodes a member of a novel protein kinase subfamily. Plant J 2001, 26(1):101 112.

28. Knight H, Trewavas AJ, Knight MR: Calcium signalling in Arabidopsis thaliana responding to drought and salinity. Plant J 1997 12(5):1067 1078

29. Chinchilla D, Shan L, He P, de Vries S, Kemmerling B: One for all: the receptor-associated kinase BAK1. Trends Plant Sci 2009, 14(10):535 541.

30. Salathia N, Lee HN, Sangster TA, Morneau K, Landry CR, Schellenberg K, Behere AS, Gunderson KL, Cavalieri D, Jander G, Queitsch C: Indel arrays: an affordable alternative for genotyping. Plant J 2007, 51(4):727 737.

31. Shao F, Golstein C, Ade J, Stoutemyer M, Dixon JE, Innes RW: Cleavage of Arabidopsis PBS1 by a bacterial type III effector. Science 2003, 301(5637):1230 1233.

32. Laluk K, Luo H, Chai M, Dhawan R, Lai Z, Mengiste T: Biochemical and genetic requirements for function of the immune response regulator BOTRYTIS-INDUCED KINASE1 in plant growth, ethylene signaling, and PAMP-triggered immunity in Arabidopsis. Plant Cell 2011, 23(8):2831 2849.

33. Veronese P, Nakagami H, Bluhm B, Abuqamar S, Chen X, Salmeron J, Dietrich RA, Hirt H, Mengiste T: The membrane-anchored BOTRYTISINDUCED KINASE1 plays distinct roles in Arabidopsis resistance to necrotrophic and biotrophic pathogens. Plant Cell 2006, 18(1):257 273.

34. Zhang J, Li W, Xiang T, Liu Z, Laluk K, Ding X, Zou Y, Gao M, Zhang X, Chen S, Mengiste T, Zhang Y, Zhou JM: Receptor-like cytoplasmic kinases integrate signaling from multiple plant immune receptors and are targeted by a Pseudomonas syringae effector. Cell Host Microbe 2010 7(4):290 301.

35. Cheng C, Gao X, Feng B, Sheen J, Shan L, He P: Plant immune response to pathogens differs with changing temperatures. Nat Commun 2013, 4:2530.

36. Li L, Li M, Yu L, Zhou Z, Liang X, Liu Z, Cai G, Gao L, Zhang X, Wang Y, Chen S, Zhou JM: The FLS2-associated kinase BIK1 directly phosphorylates the NADPH Oxidase RbohD to control plant immunity. Cell Host Microbe 2014, 15(3):329 338

37. Guy E, Lautier M, Chabannes M, Roux B, Lauber E, Arlat M, Noel LD: xopACtriggered immunity against Xanthomonas depends on Arabidopsis receptor-like cytoplasmic kinase genes PBL2 and RIPK. PLOS One 2013 8(8):e73469.

38. Lu D, Wu S, Gao X, Zhang Y, Shan L, He P: A receptor-like cytoplasmic kinase, BIK1, associates with a flagellin receptor complex to initiate plant innate immunity. Proc Natl Acad Sci U S A 2010, 107(1):496 501.

39. Lin W, Lu D, Gao X, Jiang S, Ma X, Wang Z, Mengiste T, He P, Shan L: Inverse modulation of plant immune and brassinosteroid signaling pathways by the receptor-like cytoplasmic kinase BIK1. Proc Natl Acad Sci U S A 2013, 110(29):12114 12119.

40. Shinya T, Yamaguchi K, Desaki Y, Yamada K, Narisawa T, Kobayashi Y, Maeda K, Suzuki M, Tanimoto T, Takeda J Nakashima M, Funama R, Narusaka M, Narusaka Y, Kaku H, Kawasaki T, Shibuya N: Selective regulation of the chitin-induced defense response by the Arabidopsis receptor-like cytoplasmic kinase PBL27. Plant J 2014, 10.1111/tpj.12535

41. Halter T, Imkampe J, Mazzotta S, Wierzba M, Postel S, Bucherl C, Kiefer C, Stahl M, Chinchilla D, Wang X, Nrnberger T, Zipfel C, Clouse S, Borst JW, 
Boeren S, de Vries SC, Tax, F, Kemmerling B: The leucine-rich repeat receptor kinase BIR2 is a negative regulator of BAK1 in plant immunity. Curr Biol 2014, 24(2):134 143.

42. Xu J, Wei X, Yan L, Liu D, Ma Y, Guo Y, Peng C, Zhou H, Yang C, Lou Z, Shui W: Identification and functional analysis of phosphorylation residues of the Arabidopsis BOTRYTIS-INDUCED KINASE1. Protein Cell 2013, 4(10):771 781.

43. Segonzac C, Macho AP, Sanmartin M, Ntoukakis V, Sanchez-Serrano JJ, Zipfel C: Negative control of BAK1 by protein phosphatase 2A during plant innate immunity. EMBO J 2014, 33(18):2069 2079.

44. Lin W, Li B, Lu D, Chen S, Zhu N, He P, Shan L: Tyrosine phosphorylation of protein kinase complex BAK1/BIK1 mediates Arabidopsis innate immunity. Proc Natl Acad Sci U S A 2014, 111(9):3632 3637.

45. Feng F, Yang F, Rong W, Wu X, Zhang J, Chen S, He C, Zhou JM: A Xanthomonas uridine $5^{\prime}$-monophosphate transferase inhibits plant immune kinases. Nature 2012, 485(7396):114 118.

46. Zhang J, Zhou JM: Plant immunity triggered by microbial molecular signatures. Mol Plant 2010, 3(5):783 793.

47. Henry E, Yadeta KA, Coaker G: Recognition of bacterial plant pathogens: local, systemic and transgenerational immunity. New Phytol 2013, 199(4):908 915.

48. Burr CA, Leslie ME, Orlowski SK, Chen I, Wright CE, Daniels MJ, Liljegren SJ: CAST AWAY, a membrane-associated receptor-like kinase, inhibits organ abscission in Arabidopsis. Plant Physiol 2011, 156(4):1837 1850

49. Stael S, Bayer RG, Mehlmer N, Teige M: Protein N-acylation overrides differing targeting signals. FEBS Lett 2011, 585(3):517 522.

50. Qi D, Dubiella U, Kim SH, Sloss DI, Dowen RH, Dixon JE, Innes RW: Recognition of the protein kinase AVRPPHB SUSCEPTIBLE1 by the disease resistance protein RESISTANCE TO PSEUDOMONAS SYRINGAE5 is dependent on s-acylation and an exposed loop in AVRPPHB SUSCEPTIBLE1. Plant Physiol 2014, 164(1):340 351.

51. Ade J, DeYoung BJ, Golstein C, Innes RW: Indirect activation of a plant nucleotide binding site-leucine-rich repeat protein by a bacterial protease. Proc Natl Acad Sci U S A 2007, 104(7):2531 2536.

52. van der Hoorn RA, Kamoun S: From guard to decoy: a new model for perception of plant pathogen effectors. Plant Cell 2008, 20(8):2009 2017.

53. Kadota Y, Sklenar J, Derbyshire P, Stransfeld L, Asai S, Ntoukakis V, Jones JD, Shirasu K, Menke F, Jones A, Zipfel C: Direct regulation of the NADPH oxidase RBOHD by the PRR-associated kinase BIK1 during plant immunity. Mol Cell 2014, 54(1):43 55.

54. Delker C, Poschl Y, Raschke A, Ullrich K, Ettingshausen S, Hauptmann V, Grosse I, Quint M: Natural variation of transcriptional auxin response networks in Arabidopsis thaliana. Plant Cell 2010, 22(7):2184 2200.

55. Lee J, Rudd JJ, Macioszek VK, Scheel D: Dynamic changes in the localization of MAPK cascade components controlling pathogenesisrelated $(P R)$ gene expression during innate immunity in parsley. J Biol Chem 2004, 279(21):22440 22448.

\section{Submit your next manuscript to BioMed Central and take full advantage of:}

$\otimes$ Convenient online submission

$\otimes$ Thorough peer review

$\bigotimes$ No space constraints or color $\Downarrow$ gure charges

$\nabla$ Immediate publication on acceptance

Q Inclusion in PubMed, CAS, Scopus and Google Scholar

$\otimes$ Research which is freely available for redistribution

Submit your manuscript at www.biomedcentral.com/submit
C Biomed Central 